\title{
A Classical Complete Action for a System of Small-Point Massive Charged Particles in General Relativity
}

\author{
Gonzalo Ares de Parga, Adriana Avalos-Vargas \\ Departamento de Física, Escuela Superior de Física yMatemáticas, Instituto Politécnico Nacional, U. P. Adolfo \\ López Mateos, Zacatenco, C.P. 07738, Mexico City, Mexico \\ Email: gadpau@hotmail.com
}

Received 23 March 2015; accepted 25 August 2015; published 28 August 2015

Copyright (C) 2015 by authors and Scientific Research Publishing Inc.

This work is licensed under the Creative Commons Attribution International License (CC BY). http://creativecommons.org/licenses/by/4.0/

c) (i) Open Access

\begin{abstract}
A classical action which describes the motion of a system of small-point massive charged particles including the existence of the electromagnetic and gravitational self-forces, Maxwell equations and Einstein field equations is presented. The action possesses the particularity of being a functional of the variables $z_{i}\left(\tau_{i}\right)$, the trajectory of the $i$-particle, $A_{\alpha}(x)$, the electromagnetic 4-potential, and $g_{\alpha \beta}(x)$, the metric tensor. It is also considered that the metric tensor $g_{\alpha \beta}(x)$ and the potential $A_{\alpha}(x)$ are not functions of the trajectory of each particle when the variations with respect to the trajectories of the particles are done. That is, the action is complete. The electromagnetic and the gravitational self-forces are analyzed.
\end{abstract}

\section{Keywords}

\section{General Relativity, Classical Electrodynamics, Self-Action}

\section{Introduction}

Classical Electrodynamics and General Relativity represent two of the most important theories developed during the last century. Although they are apparently formally consistent and closed models, there still exist some unsolved issues like the electromagnetic and gravitational self-forces. Indeed, even if for many authors the Lorentz-Dirac equation [1] represents the best description of the motion of a charged point particle in Special Relativity, different proposals have appeared in order to avoid the unphysical predictions of such equation as the runaway solutions and the preaccelerations [2]. Nowadays, many authors consider the Landau-Lifshitz equation of motion as an excellent approximation which prevents questionable solutions due to its second order equation 
characteristic [3]-[7]. On the other hand, Yaghyian [8] supports the Lorentz-Dirac equation basically arguing that due to the structure of the charge, the problematic aspects of the equation can be put on a side. The LorentzDirac equation can be generalized to General Relativity by using the Principle of Equivalence but the result fails to coincide with the one obtained by generalizing Dirac's method to General Relativity [9] [10]. Two new terms appear, one geometrical, Ricci term, and the other depending on the past of the particle, the tail term, making unworkable the principle of equivalence for charged particles. However, some authors still defend the Principle of Equivalence [11] [12], contributing to enlarging the discussion about the equation of motion of a charged particle in General Relativity [13]. However, different calculations done applying distinct methods lead to the same Hobbs equation. Indeed, López-Bonilla et al. [14] among others, by using Teitelboim-Plebañski average technique [15] [16], obtained the Hobbs equation. A very formal deduction has been exposed by Poisson, Pound and Vega [17] where different techniques and method are used in order to deduce the equation. The method used Hadamard's Green solutions [18] and Detweiler technique [19] [17] to define a regular Green solution avoiding the mass renormalization. This formal deduction makes very conceivable the Hobbs equation. Moreover, applying similar arguments for obtaining the Landau-Lifshitz equation, Hobbs equation can be modified in order to get a Landau-Lifshitz like equation in General Relativity where the geometrical and tail terms are recovered [20].

On the other hand, using a similar method for the electromagnetic case, Mino [21] and Quinn and Wald [20], among others, obtained an equation which described the gravitational self-force of a non charged particle submitted to a background (vacuum) gravitational field, the MiSaTaQuWa equation. Moreover, a renormalization process can be avoided also by using Detweiler technique [19], as it has been shown by Poisson et al. [17]. However, a general equation of motion which includes the electromagnetic and gravitational self-forces, for a charged particle interacting with other particles, seems to be very difficult to obtain due to the non-linearity of the equations in General Relativity.

The idea of a small-point charged massive particle may be confusing, since in general a point particle is represented as a delta function and generates divergences. In fact, it behaves as a black hole. Therefore, it is necessary to represent the point particle as an extended body. However, as Wald and Quinn [20] have noticed, an extended body will presumably collapse to a black hole before a point particle limit can be achieved. Then, it has to be considered that the size, the charge and the mass of the particle are sufficiently small compared with the scale of the variation of the electromagnetic and gravitational background fields, and the self-field effects do not become dominant but are not negligible. Finally, although some attempts have been made to propose an internal structure of the electron [22], a small-point particle will be represented by using a Delta function but with the constraint described above.

The motion of charged particles are described by adding to the Lorentz force, the self-force. The Lorentz force can also be obtained by proposing an action with an external field [23], but the self-force has to be added too. Moreover, an action for a single charged can also be considered leading to the self-force term deduced by Hobbs [17]. In a similar way, by considering a background gravitational field (vacuum), a gravitational selfforce is obtained, the MiSaTaQuWa equation. However, when a general action is considered in order to obtain equations of motions for charged particles, Maxwell equations and Einstein field equations, the action has to be separately treated. Indeed, first the action is varied as functional of the trajectories, where the potentials $A_{\alpha}$ and the metric tensor $g_{\alpha \beta}$ are taken as functions of the trajectories $z_{i}$ of each particles, then for obtaining the Maxwell equations and the Einstein field equations, the potentials $A_{\alpha}$ and the metric tensor $g_{\alpha \beta}$ are considered as functions of the 4-vector coordinate, $x$, of the 4-volume [24]. Moreover, in this scheme, the electromagnetic and gravitational self-forces are hidden. Finally, we can support Hammond's idea that the deduction of the reaction term is quite artificial. Indeed, citing Hammond [25]: "In other words, it is not assumed a priori that a self-interactions exists, it is not put into the action principle from which the equations are derived, it is a consequence of the theory. This result, that self-interactions are not included in the basic formulation of theory, has been called a formal inconsistency in the theory". The purpose of this article consists of proposing a complete functional action for a system of charged small-point particles which is stationary with respect to small variations of the trajectories, $z_{i}$, the potentials, $A_{\alpha}(x)$, and the metric tensor, $g_{\alpha \beta}(x)$, leading to the equations of motion for each particle including the self-forces, both electromagnetic and gravitational, Maxwell equations and Einstein field equations. It has to be noticed that the existences of the self-forces are formally obtained but their expressions are not deduced. Is worth noting that the obtained action is original because normally in the particle and interaction actions, the mistake of writing both actions as functions of the particle trajectories is committed. 
At first glance, one might think that the system of equations, including the self-forces, makes no sense because the electromagnetic field and the metric tensor diverge at the particle world lines. However, as we have noted above, the different techniques developed to obtain an expression for the self-forces avoid the divergences by using renormalization processes as Dirac [1] did or by considering regular Greens functions as Detweiler did [17] [19], among others. In both cases, an average of a regular field (not divergent) over a small surface is accomplished in order to calculate the self-force; in this order of ideas the concept of small-point massive charged particle is consistent with the technique cited above. Moreover, as we mentioned before, the use of the point character of the charged particles represents just a ruse due to the unknowledge of the structure of the charges. Moreover, a technique developed by Tessarotto et al. [26], by using a wire function which can be identified with a suitable distribution of the charge, may avoid the divergences. Or simply, as an axiomatic approach, in order to avoid the divergences, it is convenient to require that the average techniques describe the self-actions on smallpoint particles.

One might ask why quantum picture is not adopted. Indeed, Galley et al. [27] provides a quantum field theoretical derivation of the Abraham-Lorentz-Dirac equation and of the MiSaTaQuWa equation. Although the approach is performed with an interaction action which contains parallel propagators, $g_{\mu}^{\alpha}\left(z_{i}, x\right)$, in the particle action the parallel propagator is not included. Indeed, in an interaction action, the stress-energy tensor expression contains the parallel propagators. For obtaining, the Einstein field equations, Poisson et al. [17] use a particle action with a metric tensor which is function of the trajectory of the particle. They claimed that the energy-stress tensor is derived by functional differentiation of the particle action with respect to metric tensor evaluated at $x$, that is $g_{\alpha \beta}(x)$. This is done by expressing $g_{\mu \nu}\left(z_{i}\right)=g_{\mu}^{\alpha} g_{\mu}^{\beta} g_{\alpha \beta}(x)$. Therefore, the purpose of the article will be accomplished by including the parallel propagators in the complete action. Quantum aspects are not considered since the goal consists of finding the form of the complete action.

Summarizing, the difference with the standard treatments consists of including the parallel propagator in the particle and interaction actions in order to obtain an action for a system of charged small-point particles which functionality does not possesses inconsistencies and the variations of each degree of freedom, $z^{i}, g_{\alpha \beta}(x)$ and $A_{\alpha}(x)$, lead to the equations of motion for each particle, the Einstein field equations and the Maxwell equations in curved space.

The article is organized as follows: in the second section, the complete functional action for a system of charged particle in curved space is proposed. The third section is advocated to obtain the formal equations of motion for each particle with self-forces, both electromagnetic and gravitational, the Maxwell equations and the Einstein field equations. In the fourth section, an analysis about the self-forces is presented. Some concluding remarks are done in the fifth section.

\section{The Complete Action}

\subsection{Least Squares Estimation and Strong Consistency}

In order to describe the motion of a charged particle submitted to an external electromagnetic field in Special Relativity, a simple action is proposed,

$$
S_{\text {extF }}=\int_{-\infty}^{\infty}\left(-m \sqrt{n_{\mu \nu} \frac{\mathrm{d} z^{\mu}}{\mathrm{d} \tau} \frac{\mathrm{d} z^{v}}{\mathrm{~d} \tau}}+q A_{\mu} \frac{\mathrm{d} z^{\mu}}{\mathrm{d} \tau}\right) \mathrm{d} \tau,
$$

where $m, n_{\mu v}, z^{\mu}, \tau, q$ and $A_{\mu}$ represent the mass of the particle, the Minkowski metric tensor, the 4-vector coordinate of the particle, the proper time of the particle, the charge of the particle and the 4-vector electromagnetic potential, respectively. By considering that the action is stationary with respect to small variations of the particle trajectory, the Lorentz force is obtained

$$
m a^{\mu}=q F^{\mu v}(z) v_{v}
$$

where $v_{v}$ and

$$
F_{\alpha \beta}=\frac{\partial A_{\beta}}{\partial x^{\alpha}}-\frac{\partial A_{\alpha}}{\partial x^{\beta}} .
$$

Obviously, no reaction force is deduced. The Maxwell equations can be derived by a similar procedure taking 
an electromagnetic action,

$$
S_{F}=\int_{V^{4}}\left(-\frac{1}{4} F^{\alpha \beta} F_{\alpha \beta}+j^{\alpha} A_{\alpha}\right) \mathrm{d} x^{4},
$$

and considering that $S_{F}$ is stationary with respect small variations of the 4-vector potential, $A_{\mu}(x)$, one obtains

$$
\frac{\partial F^{\mu \nu}}{\partial x^{\nu}}=j^{\mu}
$$

where $j^{\mu}$ is the 4-vector density. A straightforward generalization to General Relativity can be done. However, it has to be noted that no reaction force or self-force is obtained and two different actions are considered. When a total Lagrangian for a system of charged particles in General Relativity is proposed [24], in order to describe a stationary situation with respect to small variations of the particle trajectories and the 4-vector potential, taken the metric tensor, $g_{\mu \nu}$, as a prescribed external gravitational field, the existence of self-interaction will be deduced in a natural way as we will see. Nevertheless, a new problem emerged. Indeed, the standard action is

$$
S_{M}=\left\{\sum_{i=1}^{n} \int_{-\infty}^{\infty} \mathrm{d} p\left(-m_{i} \sqrt{-g_{\mu \nu}\left(z_{i}\right) \frac{\mathrm{d} z_{i}^{\mu}}{\mathrm{d} p} \frac{\mathrm{d} z_{i}^{v}}{\mathrm{~d} p}}+q_{i} A_{\mu}\left(z_{i}\right) \frac{\mathrm{d} z_{i}^{\mu}}{\mathrm{d} p}\right)\right\}-\int \mathrm{d} x^{4} \frac{1}{4} \sqrt{-g} F^{\mu \nu}(x) F_{\mu \nu}(x),
$$

where $p, m_{i}, g_{\mu v}, z_{i}^{\mu}$ and $q_{i}$ represent any parameter, the mass of the $i$-particle, the metric tensor and the charge of the $i$-particle, respectively. Making the variation with respect to $z_{i}$ and $A_{\mu}(x)$, the Lorentz force and the Maxwell equations in General Relativity are deduced. However, when the variation with respect the 4-potential, $A_{\mu}(x)$, is done, $A_{\mu}$ in the interacting term is considered as a function of the position $x$, and the Maxwell equations are obtained; that is, the 4-potential $A$ is considered as a function of $z_{i}, \delta A_{\mu}\left(z_{i}\right)$, for deducing the equations of motion of the charged particles and as a function of $x, \delta A_{\mu}(x)$, for deducing the Maxwell equations depending on the convenient. Therefore, the result is obtained by misunderstanding the concept of functionality of an action at the moment of obtaining the variation with respect the variables. in this sense, if $z_{i}$ and $A_{\mu}(x)$ represent the variables of the action, they must be considered as independent and therefore, the variation of one of them implies that the other one must be kept as a constant during this process. Finally, the standard action should be abandoned for a new one that considers independent variables; that is: the new action must be written as $S_{P F}=S_{P F}\left(z_{i}, A(x)\right)$, which represents the appropriate form for the required variations and not as $S_{P F}=S_{P F}\left(z_{i}, A\left(z_{i}\right)\right)$.

\subsection{Parallel Propagator}

In order to be able to propose a complete action it is necessary to define some mathematical tools. Let us consider an orthonormal basis, a tetrad $e_{i a}^{\mu}\left(z_{i}\left(p_{o}\right)\right)$ that is transported in parallel along the trajectory of the $i$ particle to each point of it $z_{i}(p)$ where $p$ represents any parameter describing the trajectory of each particle and $p_{o}$ is a particular value of $p$. That is: from $p_{o}$, the tetrad $e_{i a}^{\mu}\left(z_{i}\left(p_{o}\right)\right)$ is transported in parallel along the trajectory of each particle obtaining $e_{i a}^{\mu}\left(z_{i}\left(p_{o}\right)\right)$. Then, in each point of the particle trajectory a tetrad $e_{i a}^{\mu}\left(z_{i}\left(p_{o}\right)\right)$ is well-defined. Then we can define the tetrad $e_{i a}^{\alpha}\left(x, z_{i}(p)\right)$ as the one obtained by parallel transport of the tetrad $e_{i a}^{\mu}\left(z_{i}\left(p_{o}\right)\right)$ from $z_{i}(p)$ to the point $x$ along the geodesic that links $z_{i}(p)$ and $x$. We assume that $x$ belongs to $\mathcal{N}\left(z_{i}\left(p_{o}\right)\right)$, the normal convex neighborhood of $z_{i}(p)$ (the set of points that are linked to $z_{i}(p)$ by a unique geodesic). Notice that $e_{i a}^{\alpha}\left(x, z_{i}(p)\right)$ may be different of the tetrad obtained in $x$ from another $p^{\prime}\left(e_{i a}^{\alpha}\left(x, z_{i}(p)\right)\right) \neq e_{i a}^{\alpha}\left(x, z_{i}\left(p^{\prime}\right)\right)$. Therefore, the bi-tensor parallel propagator is defined as

$$
g_{i \mu}^{\alpha}\left(x, z_{i}(p)\right)=e_{i a}^{\alpha}\left(x, z_{i}(p)\right) e_{i \mu}^{a}\left(z_{i}(p)\right) .
$$

The subscript " $i$ " has been introduced for defining each tetrad for each particle. The uniqueness of $g_{i \mu}^{\alpha}\left(x, z_{i}(p)\right)$ has been proved by Dewitt and Brehme [9] in the sense that it is independent of the choice of the tetrad but it does depend on $x$ and $z_{i}(p)$. A 4-vector $B^{\mu}$ or a 4-vector $D_{\alpha}$ will be transported to a point $x$ from $z_{i}(p)$ or to $z_{i}(p)$ from $x$ and both will depend on $z_{i}(p)$ and on $x$, respectively; that is: 


$$
\begin{aligned}
& B^{\alpha}\left(x, z_{i}(p)\right)=B_{p}^{\alpha}\left(x, z_{i}\right)=g_{i \mu}^{\alpha}\left(x, z_{i}(p)\right) B^{\mu}\left(z_{i}(p)\right) \\
& D_{\mu}\left(z_{i}(p), x\right)=D_{x \mu}\left(z_{i}(p)\right)=g_{i \mu}^{\alpha}\left(x, z_{i}(p)\right) D_{\alpha}(x) \text { and } \\
& C^{\alpha \beta}\left(x, z_{i}(p)\right)=C_{p}^{\alpha \beta}\left(x, z_{i}\right)=g_{i \mu}^{\alpha}\left(x, z_{i}(p)\right) g_{i v}^{\beta}\left(x, z_{i}(p)\right) C^{\mu v}\left(z_{i}(p)\right) \text { etc. }
\end{aligned}
$$

$B_{p}^{\alpha}\left(x, z_{i}\right), D_{x \mu}\left(z_{i}(p)\right)$ and $C_{p}^{\alpha \beta}\left(x, z_{i}\right)$ represent simplified notations of $B^{\alpha}\left(x, z_{i}(p)\right), D_{\mu}\left(z_{i}(p), x\right)$ and $C^{\alpha \beta}\left(x, z_{i}(p)\right)$. It has to be noted that even if the tetrads are well-defined just in the $\mathcal{N}\left(z_{i}(p)\right)$, their use is justified by noticing that a stationary situation contemplates small variations of the trajectories of the particles. Therefore, the possible non-unicity of the geodesic will be avoided. On the other hand, the variation of the metric tensor implies a variation of the tetrad. However, the variation of the tetrad, as we will see later, can be always expressed as a variation of the metric tensor and consequently it will not explicitly appears. In order to simplify the notation the subscript " $i$ " could be dropped and the tetrad and the parallel propagator could be simply expressed by

$$
\begin{aligned}
& e_{i a}^{\alpha}\left(x, z_{i}(p)\right)=e_{a}^{\alpha}\left(x, z_{i}(p)\right)=e_{p a}^{\alpha}\left(x, z_{i}\right), \quad e_{i \mu}^{a}\left(z_{i}(p)\right)=e_{\mu}^{a}\left(z_{i}(p)\right)=e_{p \mu}^{a}\left(z_{i}\right), \\
& g_{i \mu}^{\alpha}\left(x, z_{i}(p)\right)=g_{\mu}^{\alpha}\left(x, z_{i}(p)\right)=e_{a}^{\alpha}\left(x, z_{i}(p)\right) e_{\mu}^{a}\left(z_{i}(p)\right)=g_{p \mu}^{\alpha}\left(x, z_{i}\right) .
\end{aligned}
$$

We shall here adopt the convention that indices taken from the letters $\alpha$ to $\kappa$ in the Greek alphabet are always associated with the point $x$, while indices taken from $\lambda$ to $\omega$ are always associated with the particle $z_{i}$. It has to be noted that when a vector or tensor field is defined in all the space, a parallel transport of it will not coincide with the value of the field in this point. As for example, if we consider the 4-vector potential $A_{\alpha}(x)$ and the parallel propagator is applied, we have $A_{x / / \mu}\left(z_{i}\right)=g_{\mu}^{\alpha}\left(x, z_{i}(p)\right) A_{\alpha}(x) \neq A_{\mu}\left(z_{i}(p)\right)$. This is because the value of the 4-potential is governed by the inhomogeneous wave equation and not by a parallel transport. This comment has to be taken on mind at the time of applying $g_{\mu}^{\alpha}\left(x, z_{i}(p)\right)$ to any set of 4-vectors or 4-tensors in the action or not.

\subsection{Complete Action}

Once the parallel propagator has been defined, invariant quantities can be introduced in the action. Indeed, the term $q_{i} A_{\mu}\left(z_{i}\right) \frac{\mathrm{d} z_{i}^{\mu}}{\mathrm{d} p}$ can be substituted by $q_{i} g_{p \mu}^{\alpha}\left(x, z_{i}\right) A_{\alpha}(x) \frac{\mathrm{d} z_{i}^{\mu}}{\mathrm{d} p}$. This last expression represents an invariant which contains a 4-potential depending on $x$. A complete action can now be defined. The action will be a functional of the following independent variables: the trajectories of each particles, $z_{i}$, the electromagnetic 4-potential, $A_{\alpha}(x)$ and the metric tensor, $g_{\alpha \beta}(x)$. Since the 4-potential and the metric tensor cannot be expressed as functions of the trajectories, the parallel propagator will appear in the complete action. The proposed action is composed by:

A- The particle action, $S_{p}$,

$$
S_{P}=\int_{V^{4}} \sqrt{-g} \mathrm{~d} x^{4} \int_{-\infty}^{\infty} \mathrm{d} p\left\{\sum_{i=1}^{n}-m_{i} \delta\left(x, z_{i}\right) \sqrt{-g_{\alpha \beta} g_{p \mu}^{\alpha}\left(x, z_{i}\right) g_{p \nu}^{\beta}\left(x, z_{i}\right) \frac{\mathrm{d} z_{i}^{\mu}}{\mathrm{d} p} \frac{\mathrm{d} z_{i}^{v}}{\mathrm{~d} p}}\right\},
$$

where $p$ represents any parameter and $\delta\left(x, z_{i}\right)=\frac{\delta\left(x-z_{i}\right)}{\sqrt{-g}}$, with $g$ the determinant of the metric tensor;

B- The interaction action, $S_{I}$,

$$
S_{I}=\int_{V^{4}} \sqrt{-g} \mathrm{~d} x^{4} \int_{-\infty}^{\infty} \mathrm{d} p\left\{\sum_{i=1}^{n} \delta\left(x, z_{i}\right) q_{i} A_{\alpha}(x) g_{p \mu}^{\alpha}\left(x, z_{i}\right) \frac{\mathrm{d} z_{i}^{\mu}}{\mathrm{d} p}\right\} ;
$$

C- The electromagnetic field action, $S_{F}$,

$$
S_{F}=-\frac{1}{4} \int_{V^{4}} \sqrt{-g} \mathrm{~d} x^{4} F^{\alpha \beta} F_{\alpha \beta},
$$


where the electromagnetic field-strength tensor $F_{\alpha \beta}$ is the same as in Equation (3);

D- The gravitational field action, $S_{G}$,

$$
S_{G}=-\frac{1}{16 \pi G} \int_{V^{4}} \sqrt{-g} \mathrm{~d} x^{4} R(x),
$$

being $R(x)$ the curvature scalar. Finally, we arrive to the complete action, $S$,

$$
\begin{aligned}
S= & S_{P}+S_{I}+S_{F}+S_{G} \\
= & \int_{V^{4}} \sqrt{-g} \mathrm{~d} x^{4}\left\{\int_{-\infty}^{\infty} \mathrm{d} p \sum_{i=1}^{n} \delta\left(x, z_{i}\right)\left\{-m_{i} \sqrt{-g_{\alpha \beta} g_{p \mu}^{\alpha}\left(x, z_{i}\right) g_{p \nu}^{\beta}\left(x, z_{i}\right) \frac{\mathrm{d} z_{i}^{\mu}}{\mathrm{d} p} \frac{\mathrm{d} z_{i}^{\nu}}{\mathrm{d} p}}+q_{i} A_{\alpha} g_{p \mu}^{\alpha}\left(x, z_{i}\right) \frac{\mathrm{d} z_{i}^{\mu}}{\mathrm{d} p}\right\}\right. \\
& \left.-\frac{1}{4} F^{\alpha \beta} F_{\alpha \beta}-\frac{1}{16 \pi G} R(x)\right\}
\end{aligned}
$$

As promised in the introduction, an action is proposed whose independent variables are $z^{\mu}, g_{\alpha \beta}$ and $A_{\alpha}$ without confusing the dependence of these, i.e.: $z^{\mu}$ is a function $p, g_{\alpha \beta}$ and $A_{\alpha}$ are functions of $x$. Therefore, the action described by Equation (14) does not represent a standard action due to the introduction of the parallel propagator not only in the interaction action but also in the particle action.

\section{The Variation of the Complete Action}

Once the action is proposed, the equations arising from them must be derived by making the variations of it with respect to $z^{\mu}, g_{\alpha \beta}$ and $A_{\alpha}$. The variation of the complete action can be expressed as $\delta S=\delta S_{P}+\delta S_{I}+\delta S_{F}+\delta S_{G}$, and each action must be varied considering the variables $z_{i}(p), A_{\alpha}(x)$ and $g_{\alpha \beta}(x)$ as independent; therefore the procedure will be as follows: first the variation with respect $g_{\alpha \beta}(x)$ will be made because it is the most complicated; second, we will vary the 4-potential $A_{\alpha}(x)$; and finally, we will repeat the procedure for $z_{i}^{\mu}(p)$. The variation with respect to one of the variables will be denoted as $\delta S_{K y}$ with $K$ being $P, I, F$ or $G$ and $y$ being $z, A$ or $g$.

\subsection{Variation with Respect to the Metric Tensor $\delta S_{g}$}

The variation of $S$ with respect to the metric tensor is, $\delta S_{g}=\delta S_{P g}+\delta S_{I g}+\delta S_{F g}+\delta S_{G g}$. Let us begin by calculating $\delta S G_{g}$,

$$
\begin{aligned}
\delta S_{G g} & =-\frac{1}{16 \pi G} \int_{V^{4}} \delta_{g}(R(x) \sqrt{-g}) \mathrm{d} x^{4} \\
& =-\frac{1}{16 \pi G} \int_{V^{4}}\left(R(x)\left(\delta_{g} \sqrt{-g}\right)+\left(\delta_{g} R\right) \sqrt{-g}\right) \mathrm{d} x^{4} .
\end{aligned}
$$

Since

$$
R=g^{\alpha \beta} R_{\alpha \beta} \Rightarrow \delta_{g} R=\left(\delta_{g} g^{\alpha \beta}\right) R_{\alpha \beta}+g^{\alpha \beta}\left(\delta_{g} R_{\alpha \beta}\right),
$$

we have

$$
\delta_{g}(R(x) \sqrt{-g})=R(x)\left(\delta_{g} \sqrt{-g}\right)+\sqrt{-g}\left(\left(\delta_{g} g^{\alpha \beta}\right) R_{\alpha \beta}+g^{\alpha \beta}\left(\delta_{g} R_{\alpha \beta}\right)\right) .
$$

Let us first analyze $\delta_{g} R_{\alpha \beta}$. Some results have to be pointed out before obtaining it. We know that

$$
R_{\alpha \beta}=R_{\alpha \delta \beta}^{\delta}=\frac{\partial \Gamma_{\alpha \delta}^{\delta}}{\partial x^{\beta}}-\frac{\partial \Gamma_{\alpha \beta}^{\delta}}{\partial x^{\delta}}+\Gamma_{\alpha \delta}^{\varepsilon} \Gamma_{\beta \varepsilon}^{\delta}-\Gamma_{\alpha \beta}^{\varepsilon} \Gamma_{\delta \varepsilon}^{\delta} .
$$

Therefore, it is necessary to notice that the variation of a covariant derivative of a 4-vector is different to the covariant derivative of the variation of a 4-vector; that is: $\left(\delta V^{\alpha}\right)_{; \beta} \neq \delta\left(V_{; \beta}^{\alpha}\right)$. In order to express the covariant form of the metric tensor variation, $\delta g^{\alpha \varepsilon}$, we use the inverse property; that is: 


$$
\begin{aligned}
& g^{\alpha \delta} g_{\delta \beta}=\delta_{\beta}^{\alpha} \Rightarrow \delta\left(g^{\alpha \delta} g_{\delta \beta}\right)=0 \\
& \Rightarrow \delta\left(g^{\alpha \delta}\right) g_{\delta \beta}+g^{\alpha \delta} \delta\left(g_{\delta \beta}\right)=0 \\
& \delta g^{\alpha \varepsilon}=\delta_{\delta}^{\varepsilon} \delta g^{\alpha \delta}=g^{\varepsilon \beta} g_{\beta \delta} \delta g^{\alpha \delta}=-g^{\varepsilon \beta} g^{\alpha \delta} \delta g_{\delta \beta} .
\end{aligned}
$$

We are now in conditions to calculate the variation of the affine connections,

$$
\delta \Gamma_{\alpha \beta}^{\delta}=\frac{1}{2}\left(\delta g^{\delta \varepsilon}\right)\left(\frac{\partial g_{\varepsilon \beta}}{\partial x^{\alpha}}+\frac{\partial g_{\varepsilon \alpha}}{\partial x^{\beta}}-\frac{\partial g_{\alpha \beta}}{\partial x^{\varepsilon}}\right)+\frac{1}{2} g^{\gamma \varepsilon}\left(\frac{\partial \delta g_{\varepsilon \beta}}{\partial x^{\alpha}}+\frac{\partial \delta g_{\varepsilon \alpha}}{\partial x^{\beta}}-\frac{\partial \delta g_{\alpha \beta}}{\partial x^{\varepsilon}}\right) .
$$

By using Equation (19), we arrive at

$$
\delta \Gamma_{\alpha \beta}^{\delta}=-\frac{1}{2} g^{\delta \gamma} g^{\kappa \varepsilon}\left(\delta g_{\gamma \kappa}\right)\left(\frac{\partial g_{\varepsilon \beta}}{\partial x^{\alpha}}+\frac{\partial g_{\varepsilon \alpha}}{\partial x^{\beta}}-\frac{\partial g_{\alpha \beta}}{\partial x^{\varepsilon}}\right)+\frac{1}{2} g^{\delta \varepsilon}\left(\frac{\partial \delta g_{\varepsilon \beta}}{\partial x^{\alpha}}+\frac{\partial \delta g_{\varepsilon \alpha}}{\partial x^{\beta}}-\frac{\partial \delta g_{\alpha \beta}}{\partial x^{\varepsilon}}\right) .
$$

Therefore, from Equations (20) and (21), we obtain that $\delta \Gamma_{\alpha \beta}^{\delta}$,

$$
\begin{aligned}
& \delta \Gamma_{\alpha \beta}^{\delta}=-g^{\delta \gamma}\left(\delta g_{\gamma \kappa}\right) \Gamma_{\alpha \beta}^{\kappa}+\frac{1}{2} g^{\delta \varepsilon}\left(\frac{\partial \delta g_{\varepsilon \beta}}{\partial x^{\alpha}}+\frac{\partial \delta g_{\varepsilon \alpha}}{\partial x^{\beta}}-\frac{\partial \delta g_{\alpha \beta}}{\partial x^{\varepsilon}}\right) \\
& \delta \Gamma_{\alpha \beta}^{\delta}=\frac{1}{2} g^{\delta \varepsilon}\left(\left(\delta g_{\varepsilon \beta}\right)_{; \alpha}+\left(\delta g_{\varepsilon \alpha}\right)_{; \beta}-\left(\delta g_{\alpha \beta}\right)_{; \varepsilon}\right),
\end{aligned}
$$

represents a tensor [24]. Therefore, the variation of $R_{\alpha \beta}$ is

$$
\delta R_{\alpha \beta}=\frac{\partial \delta \Gamma_{\alpha \delta}^{\delta}}{\partial x^{\beta}}-\frac{\partial \delta \Gamma_{\alpha \beta}^{\delta}}{\partial x^{\delta}}+\left(\delta \Gamma_{\alpha \delta}^{\varepsilon}\right) \Gamma_{\beta \varepsilon}^{\delta}+\Gamma_{\alpha \delta}^{\varepsilon}\left(\delta \Gamma_{\beta \varepsilon}^{\delta}\right)-\left(\delta \Gamma_{\alpha \beta}^{\varepsilon}\right) \Gamma_{\delta \varepsilon}^{\delta}-\Gamma_{\alpha \beta}^{\varepsilon}\left(\delta \Gamma_{\delta \varepsilon}^{\delta}\right)=\left(\delta \Gamma_{\alpha \delta}^{\delta}\right)_{; \beta}-\left(\delta \Gamma_{\alpha \beta}^{\delta}\right)_{; \delta} .
$$

So, the last term of Equation (17) can be written as

$$
\sqrt{-g} g^{\alpha \beta} \delta R_{\alpha \beta}=\sqrt{-g}\left(g^{\alpha \beta}\left(\delta \Gamma_{\alpha \delta ; \beta}^{\delta}\right)-g^{\alpha \beta}\left(\delta \Gamma_{\alpha \beta ; \delta}^{\delta}\right)\right) .
$$

On the other hand, since $V_{; \alpha}^{\alpha}=\frac{1}{\sqrt{-g}} \frac{\partial\left(\sqrt{-g} V^{\alpha}\right)}{\partial x^{\alpha}}$, we obtain

$$
\sqrt{-g} g^{\alpha \beta} \delta R_{\alpha \beta}=\frac{\partial}{\partial x^{\beta}}\left(\sqrt{-g} g^{\alpha \beta} \delta \Gamma_{\alpha \delta}^{\delta}\right)-\frac{\partial}{\partial x^{\delta}}\left(\sqrt{-g} g^{\alpha \beta} \delta \Gamma_{\alpha \beta}^{\delta}\right) .
$$

This term vanishes when it is integrated over the 4-volume. Returning to Equation (17), it is clear that we need to know $\delta \sqrt{-g}$. It is easy to see that

$$
\delta \sqrt{-g}=\frac{1}{2} \frac{\delta(-g)}{\sqrt{-g}}=\frac{1}{2} \sqrt{-g} g^{\alpha \beta} \delta g_{\alpha \beta} .
$$

By using Equations (19) and (26), the variation of $S_{G}$ with respect to the metric tensor, $\delta S_{G g}$, Equation (15), can be expressed as:

$$
\begin{aligned}
\delta S_{G g} & =-\frac{1}{16 \pi G} \int_{V^{4}}\left(R(\delta \sqrt{-g})+\sqrt{-g}\left(\left(\delta g^{\alpha \beta}\right) R_{\alpha \beta}+g^{\alpha \beta}\left(\delta R_{\alpha \beta}\right)\right)\right) \mathrm{d} x^{4} \\
& =\frac{1}{16 \pi G} \int_{V^{4}} \sqrt{-g}\left(R^{\alpha \beta}-\frac{1}{2} R g^{\alpha \beta}\right) \delta g_{\alpha \beta} \mathrm{d} x^{4} .
\end{aligned}
$$

It is clear that if we consider an empty space, it is obtained $R^{\alpha \beta}-\frac{1}{2} \operatorname{Rg}^{\alpha \beta}=0$, which represent the Einstein field equations in vacuum. However, if the system contains point massive charged particles, the other parts of the action have to be considered. Let us now calculate the variation of $S_{P}$, Equation (10) with respect to the metric tensor; that is: $\delta S_{P g}$. A simple inspection shows that it will be necessary to know the variation of the 
parallel propagator with respect to the metric tensor. Indeed, when the metric tensor suffers a variation, the tetrads, which generate the parallel propagators, are also varied and consequently the parallel propagators too. Then, we need to know the relation between $\delta g_{\alpha \beta}, \delta e_{p a}^{\alpha}$ and $\delta g_{p \mu}^{\alpha}$.

$$
\begin{aligned}
& g_{\alpha \beta} e_{p a}^{\alpha} e_{p b}^{\beta}=n_{a b} \Rightarrow \delta\left(g_{\alpha \beta} e_{p a}^{\alpha} e_{p b}^{\beta}\right)=0 \Rightarrow \\
& \delta\left(g_{\alpha \beta}\right) e_{p a}^{\alpha} e_{p b}^{\beta}=-g_{\alpha \beta}\left(\delta e_{p a}^{\alpha}\right) e_{p b}^{\beta}-g_{\alpha \beta} e_{p a}^{\alpha}\left(\delta e_{p b}^{\beta}\right)=-g_{\alpha \beta}\left(e_{p b}^{\beta} \delta e_{p a}^{\alpha}+e_{p a}^{\alpha} \delta e_{p b}^{\beta}\right) .
\end{aligned}
$$

In the other side,

$$
\begin{aligned}
\delta g_{\delta \varepsilon} & =\delta_{\delta}^{\alpha} \delta_{\varepsilon}^{\beta} \delta g_{\alpha \beta}=e_{p \delta}^{a} e_{p a}^{\alpha} e_{p \varepsilon}^{b} e_{p b}^{\beta} \delta g_{\alpha \beta}=e_{p \delta}^{a} e_{p \varepsilon}^{b}\left(e_{p a}^{\alpha} e_{p b}^{\beta} \delta g_{\alpha \beta}\right) \\
& =-e_{p \delta}^{a} e_{p \varepsilon}^{b} g_{\alpha \beta}\left(e_{p b}^{\beta} \delta e_{p a}^{\alpha}+e_{p a}^{\alpha} \delta e_{p b}^{\beta}\right)=-\left(e_{p \varepsilon}^{b} e_{p b}^{\beta}\right) g_{\alpha \beta} e_{p \delta}^{a} \delta e_{p a}^{\alpha}-\left(e_{p \delta}^{a} e_{p a}^{\alpha}\right) g_{\alpha \beta} e_{p \varepsilon}^{b} \delta e_{p b}^{\beta} \\
& =-\delta_{\varepsilon}^{\beta} g_{\alpha \beta} e_{p \delta}^{a} \delta e_{p a}^{\alpha}-\delta_{\delta}^{\alpha} g_{\alpha \beta} e_{p \varepsilon}^{b} \delta e_{p b}^{\beta}=-g_{\alpha \varepsilon} e_{p \delta}^{a} \delta e_{p a}^{\alpha}-g_{\delta \beta} e_{p \varepsilon}^{b} \delta e_{p b}^{\beta}=-\left(g_{\delta \alpha} e_{p \varepsilon}^{a}+g_{z \alpha} e_{p \delta}^{a}\right) \delta e_{p a}^{\alpha} .
\end{aligned}
$$

Finally, as an example, the variation of $S_{G}$ with respect to tetrad $e_{p a}^{\alpha}, \delta S_{G e}$, can be calculated. That is, we know that

$$
\begin{aligned}
\delta S_{G e} & =\frac{1}{16 \pi G} \int_{V^{4}} \sqrt{-g}\left(R^{\alpha \beta}-\frac{1}{2} R g^{\alpha \beta}\right) \delta g_{\alpha \beta} \mathrm{d} x^{4} \\
& =-\frac{1}{16 \pi G} \int_{V^{4}} \sqrt{-g}\left(R^{\alpha \beta}-\frac{1}{2} R g^{\alpha \beta}\right)\left(g_{\alpha \delta} e_{p \beta}^{a}+g_{\beta \delta} e_{p \alpha}^{a}\right) \delta e_{p a}^{\delta} \mathrm{d} x^{4} \\
& =-\frac{1}{16 \pi G} \int_{V^{4}} \sqrt{-g}\left\{R_{\delta}^{\beta} e_{p \beta}^{a} \delta e_{p a}^{\delta}+R_{\delta}^{\alpha} e_{p \alpha}^{a} \delta e_{p a}^{\delta}-\frac{1}{2} R \delta_{\delta}^{\beta} e_{p \beta}^{a} \delta e_{p a}^{\delta}-\frac{1}{2} R \delta_{\delta}^{\alpha} e_{p \alpha}^{a} \delta e_{p a}^{\delta}\right\} \mathrm{d} x^{4} \\
& =-\frac{1}{8 \pi G} \int_{V^{4}} \sqrt{-g}\left(R_{\delta}^{\beta}-\frac{1}{2} R \delta_{\delta}^{\beta}\right) e_{p \beta}^{a} \delta e_{p a}^{\delta} \mathrm{d} x^{4} .
\end{aligned}
$$

The result described by Equation (27) has been recovered. It has to be remembered that the use of the tetrad is just justified in the $\mathcal{N}\left(z_{i}\right)$. This is satisfied since a stationary situation contemplates small variations of the trajectories of the particles. Let us return to the variation $\delta S_{P}$ with respect to the metric tensor, $\delta S_{P g}$. If we analyze Equation (10), it can be observed that the important term to be varied is $g_{\alpha \beta}(x) g_{\mu}^{\alpha}\left(x, z_{i}\right) g_{v}^{\beta}\left(x, z_{i}\right)$. However, the variation with respect to the metric tensor is related with the one respect to the tetrad by Equation (29). It is necessary to investigate the variation of the parallel propagator with respect to the tetrad,

$$
\delta g_{p \mu}^{\alpha}\left(x, z_{i}\right)=\left(\delta e_{p a}^{\alpha}(x)\right) e_{p \mu}^{a}\left(z_{i}\right)+e_{p a}^{\alpha}(x)\left(\delta e_{p \mu}^{a}\left(z_{i}\right)\right) .
$$

The first variation, $\delta e_{p a}^{\alpha}(x)$, of the right term is natural and it does not represent any problem. However, it is necessary to analyze the second variation $\delta e_{p \mu}^{a}\left(z_{i}\right)$ from a conceptual point of view. Indeed, the meaning is $\delta e_{p \mu}^{a}\left(z_{i}\right)=\left(\delta e_{p \mu}^{a}(x)\right)_{x=z_{i}}$. However, when $\delta g_{p \mu}^{\alpha}\left(x, z_{i}\right)$ is preceded by a Delta function, the integration over the 4 -volume will lead to the same result as considering it as $\delta g_{\mu}^{\alpha}\left(z_{i}, z_{i}\right)=\delta_{\mu}^{\alpha}$. That is:

$$
\int \delta\left(x-z_{i}\right) \delta g_{p \mu}^{\alpha}\left(x, z_{i}\right) f\left(x, z_{i}\right) \mathrm{d} x^{4}=\int \delta\left(x-z_{i}\right) \delta g_{p \mu}^{\alpha}\left(z_{i}, z_{i}\right) f\left(x, z_{i}\right) \mathrm{d} x^{4}=0 .
$$

Therefore, we arrive at

$$
\delta\left(g_{\alpha \beta}(x) g_{p \mu}^{\alpha}\left(x, z_{i}\right) g_{p v}^{\beta}\left(x, z_{i}\right)\right)=\left(\delta g_{\alpha \beta}(x)\right) g_{p \mu}^{\alpha}\left(x, z_{i}\right) g_{p v}^{\beta}\left(x, z_{i}\right) .
$$

By using Equation (29), we obtain (simplifying $\left.g_{p \mu}^{\alpha}\left(x, z_{i}\right)=g_{p \mu}^{\alpha}\right)$

$$
\delta\left(g_{\alpha \beta}(x) g_{p \mu}^{\alpha} g_{v}^{\beta}\right)=-\left(g_{\alpha \delta} e_{p \beta}^{a}+g_{\beta \delta} e_{p \alpha}^{a}\right)\left(\delta e_{p a}^{\delta}\right) g_{p \mu}^{\alpha} g_{p v}^{\beta} .
$$

Finally, noticing that $\sqrt{-g}$ disappears when the product with $\delta\left(x, z_{i}\right)$ is considered, we have 


$$
\begin{aligned}
& \delta S_{P g}=\int_{V^{4}} \sqrt{-g} \mathrm{~d} x^{4} \int_{-\infty}^{\infty} \mathrm{d} p \sum_{i=1}^{n} \delta\left(x, z_{i}\right)\left\{-m_{i} \delta \sqrt{-g_{\alpha \beta}(x) g_{p \mu}^{\alpha}\left(x, z_{i}\right) g_{p v}^{\beta}\left(x, z_{i}\right) \frac{\mathrm{d} z_{i}^{\mu}}{\mathrm{d} p} \frac{\mathrm{d} z_{i}^{v}}{\mathrm{~d} p}}\right\} \\
& =\int_{V^{4}} \sqrt{-g} \mathrm{~d} x^{4} \int_{-\infty}^{\infty} \mathrm{d} p \sum_{i=1}^{n} \delta\left(x, z_{i}\right)\left\{-m_{i} \frac{g_{\alpha \delta} e_{p \beta}^{a}+g_{\beta \delta} e_{p \alpha}^{a}}{2 \sqrt{-g_{\mu \nu}\left(z_{i}\right) \frac{\mathrm{d} z_{i}^{\mu}}{\mathrm{d} p} \frac{\mathrm{d} z_{i}^{v}}{\mathrm{~d} p}}}\right\}\left(\delta e_{p a}^{\delta}\right) g_{p \mu}^{\alpha} g_{p \nu}^{\beta} \frac{\mathrm{d} z_{i}^{\mu}}{\mathrm{d} p} \frac{\mathrm{d} z_{i}^{v}}{\mathrm{~d} p} \\
& =\int_{V^{4}} \sqrt{-g} \mathrm{~d} x^{4} \int_{-\infty}^{\infty} \mathrm{d} p \sum_{i=1}^{n} \delta\left(x, z_{i}\right)\left\{m_{i} \frac{g_{p \mu}^{\alpha} g_{p v}^{\beta}}{2 \sqrt{-g_{\mu v}\left(z_{i}\right) \frac{\mathrm{d} z_{i}^{\mu}}{\mathrm{d} p} \frac{\mathrm{d} z_{i}^{v}}{\mathrm{~d} p}}} \frac{\mathrm{d} z_{i}^{\mu}}{\mathrm{d} p} \frac{\mathrm{d} z_{i}^{v}}{\mathrm{~d} p}\right\} \delta g_{\alpha \beta} .
\end{aligned}
$$

It has to be noticed that we have used the identity, $g_{\alpha \beta}(x) g_{\mu}^{\alpha} g_{v}^{\beta}=g_{\mu v}\left(z_{i}\right)$, which is allowed once the variation is done. By introducing the mechanical energy-momentum, $T_{M}^{\alpha \beta}$, we can write,

$$
\delta S_{P g}=\frac{1}{2} \int_{V^{4}} \sqrt{-g} \mathrm{~d} x^{4} T_{M}^{\alpha \beta} \delta g_{\alpha \beta},
$$

where, by changing the parameter $p$ to the proper time of each particle, $\tau_{i}$,

$$
\begin{aligned}
T_{M}^{\alpha \beta} & =\int_{-\infty}^{\infty} \mathrm{d} p \sum_{i=1}^{n} \delta^{4}\left(x, z_{i}\right)\left\{m_{i} \frac{g_{\mu}^{\alpha}\left(x, z_{i}\right) g_{v}^{\beta}\left(x, z_{i}\right) \frac{\mathrm{d} z_{i}^{\mu}}{\mathrm{d} p} \frac{\mathrm{d} z_{i}^{v}}{\mathrm{~d} p}}{\sqrt{-g_{\mu \nu}\left(z_{i}\right) \frac{\mathrm{d} z_{i}^{\mu}}{\mathrm{d} p} \frac{\mathrm{d} z_{i}^{v}}{\mathrm{~d} p}}}\right\} \\
& =\sum_{i=1}^{n} \delta^{4}\left(x, z_{i}\right) \int_{-\infty}^{\infty} \mathrm{d} \tau_{i}\left\{m_{i} g_{\tau_{i} \mu}^{\alpha}\left(x, z_{i}\right) g_{\tau_{i^{v}}}^{\beta}\left(x, z_{i}\right) \frac{\mathrm{d} z_{i}^{\mu}}{\mathrm{d} \tau_{i}} \frac{\mathrm{d} z_{i}^{v}}{\mathrm{~d} \tau_{i}}\right\} .
\end{aligned}
$$

It has to be remarked that, by using Equations (27) and (36), we obtain the Einstein field equations without charges, $R^{\alpha \beta}-\frac{1}{2} \operatorname{Rg}^{\alpha \beta}+8 \pi G T_{M}^{\alpha \beta}=0$. It has to be pointed out that we would obtain the same result by making the variation with respect to the tetrads, Equation (30), and substituting Equation (29) in Equation (36). However, in the general case of a system of point massive charged particles, the total energy-momentum tensor, where the effect of the charges is included, has to be considered. That is, the variations of the interaction action, $S_{I}$, and the field action, $S_{F}$, must be done. Let us continue calculating the variation of $S_{I}$, Equation (11). It is observed that since the variation of the parallel propagator vanishes when is preceded by a Delta function, then $\delta S_{I g}=0$. On the other hand, in order to make the variation of the field action with respect to the tensor metric, we need to notice that the metric tensor is hidden in the contravariant expression of the strength tensor, $F^{\alpha \beta} F_{\alpha \beta}=g^{\alpha \delta} g^{\beta \varepsilon} F_{\delta \varepsilon} F_{\alpha \beta}$. Making use of Equation (19),

$$
\begin{aligned}
\left(\delta F^{\alpha \beta}\right) F_{\alpha \beta} & =\left(-F^{\alpha \kappa} g^{\beta \eta} \delta g_{\eta \kappa}+F^{\beta \eta} g^{\alpha \kappa} \delta g_{\eta \kappa}\right) F_{\alpha \beta} \\
& =\left(-F^{\alpha \kappa} F_{\alpha}{ }^{\eta}-F^{\beta \eta} F_{\beta}{ }^{\kappa}\right) \delta g_{\eta \kappa}=-2 F_{\alpha}{ }^{\eta} F^{\alpha \kappa} \delta g_{\eta \kappa} .
\end{aligned}
$$

Noticing that the Delta function does not appears in $S_{F}$ and using Equation (26), we arrive at

$$
\delta S_{F g}=\frac{1}{2} \int_{V^{4}} \sqrt{-g} \mathrm{~d} x^{4}\left\{F_{\alpha}{ }^{\varepsilon} F^{\alpha \kappa}-\frac{1}{4} F^{\alpha \beta} F_{\alpha \beta} g^{\varepsilon \kappa}\right\} \delta g_{\varepsilon \kappa} .
$$

Introducing the electromagnetic energy-momentum, $T_{F}^{\alpha \beta}$,

$$
\delta S_{F g}=\frac{1}{2} \int_{V^{4}} \sqrt{-g} \mathrm{~d} x^{4} T_{F}^{\alpha \beta} \delta g_{\alpha \beta},
$$


with

$$
T_{F}^{\alpha \beta}=\left\{F_{\alpha}{ }^{\eta} F^{\alpha \beta}-\frac{1}{4} g^{\alpha \beta} F_{\delta \varepsilon} F^{\delta \varepsilon}\right\} .
$$

Finally,

$$
R^{\alpha \beta}-\frac{1}{2} \operatorname{Rg}^{\alpha \beta}=-8 \pi G T^{\alpha \beta}=-8 \pi G\left(T_{M}^{\alpha \beta}+T_{F}^{\alpha \beta}\right) .
$$

This last expression represents the Einstein field equations for a system of point massive charged particles.

\subsection{Variation with Respect to the Trajectories $\delta S_{Z}$}

The variation of the complete action with respect the metric tensor has been achieved. The next step consists on calculating the variation of $S$ with respect to the trajectories of the particles. If we analyze $S_{G}$ and $S_{F}$, we will noticed that the variation with respect to the trajectories vanishes since it does not depend on the trajectories. That is: $\delta S_{G z}=\delta S_{F z}=0$. Then, before calculating $\delta S_{P z}$, let us notice the following results. Since

$$
\int_{-\infty}^{\infty} \mathrm{d} x\left(\frac{\partial}{\partial a} \delta(x-a)\right) f(x, a)=-\int_{-\infty}^{\infty} f(x, a)\left(\frac{\partial}{\partial x} \delta(x-a)\right),
$$

the variation of the Delta function will lead to

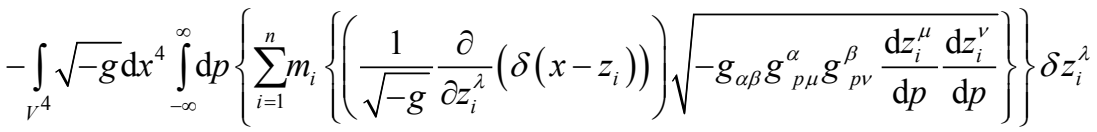

$$
\begin{aligned}
& =\int_{V^{4}} \mathrm{~d} x^{4} \int_{-\infty}^{\infty} \mathrm{d} p\left\{\sum_{i=1}^{n} m_{i}\left\{\left(\frac{\partial}{\partial x^{\lambda}} \delta\left(x-z_{i}\right)\right) \sqrt{-g_{\alpha \beta} g_{p \mu}^{\alpha} g_{p \nu}^{\beta} \frac{\mathrm{d} z_{i}^{\mu}}{\mathrm{d} p} \frac{\mathrm{d} z_{i}^{\nu}}{\mathrm{d} p}}\right\}\right\} \delta z_{i}^{\lambda} \\
& =\int_{V^{4}} \sqrt{-g} \mathrm{~d} x^{4} \int_{-\infty}^{\infty} \mathrm{d} p\left\{\sum_{i=1}^{n} \frac{1}{2} \frac{m_{i} \delta\left(x, z_{i}\right) \frac{\mathrm{d} z_{i}^{\mu}}{\mathrm{d} p} \frac{\mathrm{d} z_{i}^{\nu}}{\mathrm{d} p}}{\sqrt{-g_{\alpha \beta} g_{p \mu}^{\alpha} g_{p \nu}^{\beta} \frac{\mathrm{d} z_{i}^{\mu}}{\mathrm{d} p} \frac{\mathrm{d} z_{i}^{v}}{\mathrm{~d} p}}}\left\{\frac{\partial g_{\alpha \beta}}{\partial x^{\lambda}} g_{p \mu}^{\alpha} g_{p v}^{\beta}+g_{\alpha \beta} \frac{\partial\left(g_{p \mu}^{\alpha} g_{p \nu}^{\beta}\right)}{\partial x^{\lambda}}\right\}\right\} z_{i}^{\lambda},
\end{aligned}
$$

where we have not respect the convention ( $\alpha \rightarrow \kappa$ for $x$, and $\lambda \rightarrow \omega$ for $z$ ) for the subscript $\lambda$ due to Equation (43). As in the case of the variation of the parallel propagator with respect to the metric tensor, Equation (32), it has to be noticed that $\delta\left(x, z_{i}\right) \frac{\partial\left(g_{p \mu}^{\alpha} g_{p \nu}^{\beta}\right)}{\partial x^{\lambda}}=0$. Adding the variation of the other terms, we arrive at

$$
\delta S_{P z}=\int_{V^{4}} \sqrt{-g} \mathrm{~d} x^{4} \int_{-\infty}^{\infty} \mathrm{d} p\left\{\sum_{i=1}^{n} \frac{m_{i} \delta\left(x, z_{i}\right)}{\sqrt{-g_{\alpha \beta} g_{p \mu}^{\alpha} g_{p \nu}^{\beta} \frac{\mathrm{d} z_{i}^{\mu}}{\mathrm{d} p} \frac{\mathrm{d} z_{i}^{v}}{\mathrm{~d} p}}}\left\{\frac{1}{2} \frac{\partial g_{\alpha \beta}}{\partial x^{\lambda}} g_{p \mu}^{\alpha} g_{p \nu}^{\beta} \frac{\mathrm{d} z_{i}^{\mu}}{\mathrm{d} p} \frac{\mathrm{d} z_{i}^{v}}{\mathrm{~d} p} g_{\alpha \beta} g_{p \mu}^{\alpha} g_{p \lambda}^{\beta} \frac{\mathrm{d} z_{i}^{\mu}}{\mathrm{d} p} \frac{\mathrm{d} \delta z_{i}^{\lambda}}{\mathrm{d} p}\right\}\right\} \delta z_{i}^{\lambda} .
$$

Once we have done the variation, $\mathrm{d} p$ can be substituted by the proper time of each particle, $\mathrm{d} \tau_{i}$, the parallel propagator can be applied and the integration over the 4-volume can be achieved. The result is

$$
\delta S_{P z}=\sum_{i=1}^{n}\left\{\int_{-\infty}^{\infty} \mathrm{d} \tau_{i} m_{i}\left\{\frac{1}{2} \frac{\partial g_{\mu v}}{\partial z_{i}^{\lambda}} \frac{\mathrm{d} z_{i}^{\mu}}{\mathrm{d} p} \frac{\mathrm{d} z_{i}^{\nu}}{\mathrm{d} p}+g_{\mu \lambda} \frac{\mathrm{d} z_{i}^{\mu}}{\mathrm{d} \tau_{i}} \frac{\mathrm{d} \delta z_{i}^{\lambda}}{\mathrm{d} \tau_{i}}\right\} \delta z_{i}^{\lambda}\right\} .
$$

By integrating by parts, we arrive at

$$
\delta S_{P z}=\sum_{i=1}^{n}\left\{\int_{-\infty}^{\infty} \mathrm{d} \tau_{i} m_{i}\left\{\frac{1}{2} \frac{\partial g_{\mu \nu}}{\partial z_{i}^{\lambda}} \frac{\mathrm{d} z_{i}^{\mu}}{\mathrm{d} p} \frac{\mathrm{d} z_{i}^{\nu}}{\mathrm{d} p}-\frac{\mathrm{d}}{\mathrm{d} \tau_{i}}\left(g_{\mu \lambda} \frac{\mathrm{d} z_{i}^{\mu}}{\mathrm{d} \tau_{i}}\right)\right\} \delta z_{i}^{\lambda}\right\} .
$$


On the other hand,

$$
\begin{aligned}
& \left(\frac{1}{2} \frac{\partial g_{\mu \nu}}{\partial z_{i}^{\lambda}}-\frac{\partial g_{\lambda v}}{\partial z_{i}^{\mu}}\right) \frac{\mathrm{d} z_{i}^{\mu}}{\mathrm{d} \tau_{i}} \frac{\mathrm{d} z_{i}^{v}}{\mathrm{~d} \tau_{i}} \\
& =\frac{1}{2}\left\{\frac{1}{2} \frac{\partial g_{\mu v}}{\partial z_{i}^{\lambda}}-\frac{\partial g_{\lambda v}}{\partial z_{i}^{\mu}}+\frac{1}{2} \frac{\partial g_{\mu v}}{\partial z_{i}^{\lambda}}-\frac{\partial g_{\lambda \mu}}{\partial z_{i}^{v}}\right\} \frac{\mathrm{d} z_{i}^{\mu}}{\mathrm{d} \tau_{i}} \frac{\mathrm{d} z_{i}^{v}}{\mathrm{~d} \tau_{i}} \\
& =\frac{1}{2}\left(\frac{\partial g_{\mu v}}{\partial z_{i}^{\lambda}}-\frac{\partial g_{\lambda v}}{\partial z_{i}^{\mu}}-\frac{\partial g_{\lambda \mu}}{\partial z_{i}^{v}}\right) \frac{\mathrm{d} z_{i}^{\mu}}{\mathrm{d} \tau_{i}} \frac{\mathrm{d} z_{i}^{v}}{\mathrm{~d} \tau_{i}}=-g_{\sigma \lambda} \Gamma_{\mu \nu}^{\sigma} \frac{\mathrm{d} z_{i}^{\mu}}{\mathrm{d} \tau_{i}} \frac{\mathrm{d} z_{i}^{v}}{\mathrm{~d} \tau_{i}}
\end{aligned}
$$

Finally,

$$
\begin{aligned}
\delta S_{P z} & =-\sum_{i=1}^{n}\left\{\int_{-\infty}^{\infty} \mathrm{d} \tau_{i} m_{i} g_{\mu \lambda}\left(\frac{\mathrm{d}^{2} z_{i}^{\mu}}{\mathrm{d} \tau_{i}^{2}}+\Gamma_{\sigma \nu}^{\mu} \frac{\mathrm{d} z_{i}^{\sigma}}{\mathrm{d} \tau_{i}} \frac{\mathrm{d} z_{i}^{\nu}}{\mathrm{d} \tau_{i}}\right) \delta z_{i}^{\lambda}\right\} \\
& =-\sum_{i=1}^{n}\left\{\int_{-\infty}^{\infty} \mathrm{d} \tau_{i} m_{i} g_{\mu \lambda}\left(\frac{D v_{i}^{\mu}}{d \tau_{i}}\right) \delta z_{i}^{\lambda}\right\},
\end{aligned}
$$

where $v_{i}^{\mu}=\mathrm{d} z_{i}^{\mu} / \mathrm{d} \tau_{i}$, which implies that when no interaction is considered between the particles, we obtain the geodesic equation for each particle, $\frac{D v_{i}^{\mu}}{d \tau_{i}}=0$. However, the interaction exists between charged particles and the Interaction action has to be taken into account. It has to be remembered that the parallel propagator will not be varied due to similar reasons as in the previous cases.

$$
\delta S_{I z}=\int_{V^{4}} \sqrt{-g} \mathrm{~d} x^{4} \int_{-\infty}^{\infty} \mathrm{d} p\left\{\sum_{i=1}^{n} \delta\left(x, z_{i}\right)\left\{q_{i} \frac{\partial A_{\alpha}(x)}{\partial x^{\lambda}} g_{p \mu}^{\alpha}\left(x, z_{i}\right) \frac{\mathrm{d} z_{i}^{\mu}}{\mathrm{d} p} \delta z_{i}^{\lambda} A_{\alpha}(x) g_{p \mu}^{\alpha}\left(x, z_{i}\right) \frac{\mathrm{d} \delta z_{i}^{\mu}}{\mathrm{d} p}\right\}\right\} .
$$

By applying the parallel propagator, integrating over the 4-volume, substituting the parameter $p$ by the proper time of each particle and integrating by parts, we arrive at

$$
\delta S_{I z}=\sum_{i=1}^{n} \int_{-\infty}^{\infty} \mathrm{d} \tau_{i} q_{i}\left\{\frac{\partial A_{\mu}}{\partial x^{\lambda}}-\frac{\partial A_{\lambda}}{\partial x^{\mu}}\right\} \frac{\mathrm{d} z_{i}^{\mu}}{\mathrm{d} \tau_{i}} \delta z_{i}^{\lambda}=\sum_{i=1}^{n} \int_{-\infty}^{\infty} \mathrm{d} \tau_{i} q_{i} F_{\lambda \mu} \frac{\mathrm{d} z_{i}^{\mu}}{\mathrm{d} \tau_{i}} \delta z_{i}^{\lambda} .
$$

Combining this last result with Equation (49), we obtain

$$
\frac{D v_{i}^{\mu}}{d \tau_{i}}=q_{i} F^{\mu v} v_{i v}
$$

which seems similar to the Lorentz equation in General Relativity. However, when the Maxwell equations in General Relativity will be derived, a rapid inspection will show that the electromagnetic strength tensor in the $i$-particle, $F^{\mu v}\left(z_{i}\right)$, will include the effect of the $i$-particle; that is: the fact that the existence of the self-forces are included in this approach, it responds to criticism of Hammond [25] on the formal inconsistency in the theory (the self-force is not put into the action principle from which the equations are derived). Although the equations for the electromagnetic field have not yet been deduced, it can be guessed that a divergence appears due to the field of particle on itself. Moreover, a rapid inspection of the Einstein field equations shows that a divergence also exist due to the own gravity. A discussion of this difficulty is postponed until an analysis about the self-forces is presented in the fourth section. It has to be remembered that the particles are not point-like but small point-like, as we mentioned in the introduction, and no divergences must appears at the moment of considering the structure of the charges. However, since the particles are represented by using a Delta function, formally the divergences exist.

\subsection{Variation with Respect to the 4-Potential, $\delta A_{\alpha}(x)$}

The next step consists of varying with respect the 4-vector potential. Since $\delta S_{G A}=\delta S_{P A}=0$, we just need to calculate $\delta S_{I A}$ and $\delta S_{F A}$. Then, 


$$
\begin{aligned}
\delta S_{I A} & =\int_{V^{4}} \sqrt{-g} \mathrm{~d} x^{4} \int_{-\infty}^{\infty} \mathrm{d} p\left\{\sum_{i=1}^{n} \delta\left(x, z_{i}\right) q_{i} g_{p \mu}^{\alpha}\left(x, z_{i}\right) \frac{\mathrm{d} z_{i}^{\mu}}{\mathrm{d} p} \delta A_{\alpha}(x)\right\} \\
& =\int_{V^{4}} \sqrt{-g} \mathrm{~d} x^{4} j^{\alpha}(x) \delta A_{\alpha}(x),
\end{aligned}
$$

where

$$
j^{\alpha}=\sum_{i=1}^{n} \int_{-\infty}^{\infty} \delta\left(x, z_{i}\right) q_{i} g_{p \mu}^{\alpha}\left(x, z_{i}\right) \mathrm{d} z_{i}^{\mu} .
$$

Let us now make the variation of the electromagnetic field action with respect to the 4-potential,

$$
\delta S_{F A}=-\int_{V^{4}} \sqrt{-g} F^{\alpha \beta}(x) \frac{\partial \delta A_{\beta}}{\partial x^{\alpha}} \mathrm{d} x^{4} .
$$

Integrating by parts, we arrive at

$$
\begin{aligned}
\delta S_{F A} & =-\int_{V^{4}} \sqrt{-g} \frac{1}{\sqrt{-g}} \frac{\partial}{\partial x^{\beta}}\left(\sqrt{-g} F^{\alpha \beta}(x)\right) \delta A_{\alpha} \mathrm{d} x^{4} \\
& =-\int_{V^{4}} \sqrt{-g} F_{; \beta}^{\alpha \beta} \delta A_{\alpha} \mathrm{d} x^{4} .
\end{aligned}
$$

Equations (53) and (56) give

$$
F_{; \beta}^{\alpha \beta}=j^{\alpha},
$$

which represent the Maxwell equations in General Relativity. By using Equation (3), we obtain

$$
\square A^{\alpha}-R_{\beta}^{\alpha} A^{\beta}=-j^{\alpha},
$$

being $\square$ the D'Alembertian and where we have used the Lorentz Gauge condition, $A_{; \alpha}^{\alpha}=0$. We can now decompose the strength tensor by noticing that we can define

$$
j_{i}^{\alpha}(x)=\int_{-\infty}^{\infty} \delta\left(x, z_{i}\right) q_{i} g_{p \mu}^{\alpha}\left(x, z_{i}\right) \mathrm{d} z_{i}^{\mu},
$$

as the current density due to the $i$-particle. Therefore, each particle generates a 4-potential due to each particle $A_{i}^{\alpha}$ such that

$$
\square A_{i}^{\alpha}-R_{\beta}^{\alpha} A_{i}^{\beta}=-j_{i}^{\alpha} \text {, or } F_{i ; \beta}^{\alpha \beta}=j_{i}^{\alpha} .
$$

Therefore, we have

$$
F^{\alpha \beta}=\sum_{i=1}^{n} F_{i}^{\alpha \beta}
$$

and consequently

$$
m_{i} \frac{D v_{i}^{\mu}}{d \tau_{i}}=q_{i} \sum_{j \neq i}^{n} F_{j}^{\mu v} v_{i v}+q_{i} F_{i}^{\mu v} v_{i v} .
$$

However, $R_{\beta}^{\alpha}$, the Ricci tensor has to be calculated by using the metric tensor which includes the knowledge of the total energy-momentum tensor. Therefore, the calculation of the electromagnetic 4-potential or the electromagnetic strength field, by using Equation (60), must be accompanied by solving Equations (52) and (42).

\subsection{Summary}

From Equations (27), (36), (40), (49), (51), (61), (62), (53) and (56), the variation of the complete action, $\delta S$, is 


$$
\begin{aligned}
\delta S= & \left\{\int_{V^{4}}\left\{\frac{1}{16 \pi G}\left(R^{\alpha \beta}-\frac{1}{2} R g^{\alpha \beta}\right)+\frac{1}{2} T_{M}^{\alpha \beta}+\frac{1}{2} T_{F}^{\alpha \beta}\right\} \sqrt{-g} \delta g_{\alpha \beta} \mathrm{d} x^{4}\right. \\
& \left.-\sum_{i=1}^{n}\left\{\int_{-\infty}^{\infty} \mathrm{d} \tau_{i}\left(m_{i} g_{\mu \lambda} \frac{D v_{i}^{\mu}}{d \tau_{i}}-q_{i}\left(\sum_{j \neq i}^{n} F_{\lambda \mu}^{j}+F_{\lambda \mu}^{i}\right) \frac{\mathrm{d} z_{i}^{\mu}}{\mathrm{d} \tau_{i}}\right) \delta z_{i}^{\lambda}\right\}-\int_{V^{4}} \sqrt{-g}\left(F_{; \beta}^{\alpha \beta}-j^{\alpha}(x)\right) \delta A_{\alpha} \mathrm{d} x^{4}\right\} .
\end{aligned}
$$

Consequently,

$$
\begin{aligned}
& \left(R^{\alpha \beta}-\frac{1}{2} \operatorname{Rg}^{\alpha \beta}\right)=-8 \pi\left(T_{M}^{\alpha \beta}+T_{F}^{\alpha \beta}\right) \\
& m_{i} \frac{D v_{i}^{\mu}}{d \tau_{i}}=q_{i}\left(\sum_{j \neq i}^{n} F_{j}^{\mu v}+F_{i}^{\mu v}\right) \frac{\mathrm{d} z_{v}^{i}}{\mathrm{~d} \tau_{i}} \\
& F_{; \beta}^{\alpha \beta}=j^{\alpha}(x)
\end{aligned}
$$

represents a set of coupling equations which describes the metric tensor in all the 4 -volume space, the equation of motion for each small-point charged massive particle and the electromagnetic field in all the 4-volume space.

\section{Analysis of the Equations}

\subsection{The Divergences}

Equation (64) come from the action, Equation (14) and not from the standard action, Equation (6). It does not represent the standard Einstein-Maxwell system since the electromagnetic self-force for each particle is contained due to the term $q_{i} F_{i}^{\mu \nu} \frac{\mathrm{d} z_{v}^{i}}{\mathrm{~d} \tau_{i}}$ and the gravitational self-force is hidden in the proper time at the world line of each particle. However, a simple analysis shows that there exist divergences coming from evaluating the electromagnetic field and the metric tensor at the world line of each particle $\left(\mathrm{d} \tau_{i}^{2}=-g_{\mu \nu}\left(z_{i}\right) \mathrm{d} z_{i}^{\mu} \mathrm{d} z_{i}^{\nu}\right.$, the metric tensor must include the gravitational and electromagnetic effects due to the own particle). At first glance, it can be thought that divergence terms must appear in the self-forces expressions. Indeed, Dirac used the so-called renormalization of the mass in order to obtain its expression for the self-force in Special Relativity. Nowadays, the technique developed by Detweiler [19] could be used in order to avoid them as it has been showed by Poisson et al. [17] in General Relativity. Therefore, care must be taken when the equation of motion is deduced, not only formally, like in Equation (64), but when an specific expression is proposed. Indeed, the so-called inconsistency of the theory noticed by Hammond [25] is partially solved in the sense that self-interactions are present in Equation (64). However, their expressions for different cases have to be calculated. Moreover, various techniques have been used in order to deduce them. The most important contribution can be attributed to Dirac [1], for the Special Relativistic case, and has been imitated by other authors in General Relativity as for example DeWitt and Brehme [9], or Hobbs [10] (who corrected DeWitt and Brehme result by including the Ricci term), among others. It highlights the work done by Poisson et al. [17], using techniques developed by Detweiler [19], in order to deduce the expressions for the self-electromagnetic force for a point massive charge with a fixed metric tensor and for the self-gravitational effect for a non-charged massive point particle in a vacuum background spacetime. However, in both cases, Special Relativity and General Relativity, the deduction of the selfinteractions are still in dispute. Indeed, there exist many different proposals [2] in Special Relativity which differ from Diracs result. Besides the difficulties in flat space, naturally inherited in General Relativity, two main objections appear when curved spaces are considered. The first one is related with the interpretation of the Equivalence Principle for charged particles and the concept of radiated energy. This discussion was first induced by Boulware [11] and continued by Singal [12]. A major contribution is due to Parrot [13] since he directly put on the table the ambiguous definition of the Equivalence Principle and the concept of radiating energy. For example, about the Equivalence Principle, he commented: "However, we do conclude that most usual formulations seem not to hold in any direct and obvious way for charged particles". The second objection consists of putting on suspicion the covariancy of the deduction of the self-interactions expressions based on a not welldefined integral of the 4-energy-momentum since it attempts the illegitimate mathematical operation of summ- 
ing vectors in different tangent spaces [28]. Moreover, for Kim [29], the MiSaTaQuWa equation [21], [17] is not generally covariant and reflects a specific choice of coordinate system and would not preserve its form under an infinitesimal coordinate transformation. Moreover, some authors claim the non-existence of solutions to the equations for point particles [30]. Therefore, the knowledge of the charge structure may represent the solution to the divergences and the expressions of the self-forces [17] [22] [31]-[34].

Despite all these arguments, It has been postulated, as a property of the small-point particle, that the self-force can be obtained by an average over a surface of the field following the particle [17]. Even if the average field diverges on the world line, it does not contribute to the net force and just the regular part of the average gives an effective force which can be considered as the self-force in both cases, electromagnetic or gravitational.

\subsection{A Single Small-Point Massive Particle in a Background Vacuum Gravitational Field}

As we mentioned before, the DeWitt and Brehme equation [9], corrected by Hobbs [10], represents the first attempt to obtain an equation of motion for a point massive charged particle in a curved space. However, for our purpose, it is preferable to start by analyzing the case of a the self-gravitational effect in a background vacuum gravitational field which tensor metric is $g_{\alpha \beta}$. By putting,

$$
\tilde{g}_{\alpha \beta}=g_{\alpha \beta}+h_{\alpha \beta},
$$

where $g_{\alpha \beta}$ and $h_{\alpha \beta}$ represent the background vacuum metric tensor and the perturbation metric tensor due to the massive non-charged particle. The Einstein tensor is expressed as

$$
\tilde{G}^{\alpha \beta}=G^{\alpha \beta}(g)+\delta G^{\alpha \beta}(g, h)+\Delta G^{\alpha \beta}(g, h),
$$

where $G^{\alpha \beta}$ represents the background Einstein tensor, $\delta G^{\alpha \beta}$ is the linearized Einstein operator and $\Delta G^{\alpha \beta}$ contains the nonlinear terms. A trace-reversed metric perturbation is defined, $\gamma^{\alpha \beta}=h^{\alpha \beta}-\frac{1}{2} g^{\alpha \beta}\left(g_{\gamma \delta} h^{\gamma \delta}\right)$ and by imposing the Lorentz gauge condition, $\gamma_{; \beta}^{\alpha \beta}=0$, a wave equation is found. After a long process where Detweiler's technique [17] [19] and the average method are included, the MiSaTaQuWa [17] [20] [21] equation is obtained; that is:

$$
\frac{D v^{\mu}}{d \tau}=-\frac{1}{2}\left(g^{\mu v}+u^{\mu} u^{v}\right)\left(2 h_{v \lambda \rho}^{\text {tail }}-h_{\lambda \rho \nu}^{\text {tail }}\right) u^{\lambda} u^{v}
$$

where $h_{\nu \lambda \rho}^{\text {tail }}$ represents the tail term which depends on all the past of the particle and $\mathrm{d} \tau=-g_{\mu \nu} \mathrm{d} z^{\mu} \mathrm{d} z^{\nu}$. It has to be remembered that $g_{\mu \nu}$ represents the metric tensor of the vacuum background gravitational field. The difficulty of calculating this term is exposed in different articles [35]-[37].

\subsection{A System of Small-Point Massive Charged Particles}

First of all, it has to be noted that Equation (67) is obtained for a vacuum background gravitational field. When a system of non-charged particles is considered, the situation is more complicated. Indeed, in order to obtain the wave equation, it has been supposed that not only there is no other particles in the point considered but in all the space. That is, the background metric tensor has to be such that the vacuum Einstein equations are satisfied in all the space; that is: $\left(R^{\alpha \beta}-\frac{1}{2} R g^{\alpha \beta}\right)=0$. However, for Gal'tsov [38] when we are dealing with Einstein space, that is such that $R_{\alpha \beta}=\lambda g_{\alpha \beta}$, the MiSaTaQuWa equation is still valid within the limits of its applicability. However, for the general case it seems to be very difficult to obtain a general expression. Nevertheless, we do not have to lose sight of that, as Messaritak [39] [40] commented, the self-force is causing the particle to move on a geodesic of the metric $\tilde{g}_{\alpha \beta}=g_{\alpha \beta}+h_{\alpha \beta}$, Equation (65). In this vein, we can always describe the equation of motion, with or without other non-charged particles, as

$$
\frac{D}{d \tilde{\tau}}\left[\frac{\mathrm{d} z^{\mu}}{\mathrm{d} \tilde{\tau}}\right]=0,
$$

where $\mathrm{d} \tilde{\tau}=-\tilde{g}_{\mu \nu} \mathrm{d} z^{\mu} \mathrm{d} z^{\nu}$. For the case of vacuum space the linearization is possible and the MiSataQuWa equation is obtained by using the different methods proposed by Mino et al. [21], Wald and Quinn [20], [17]. It 
has to be remembered that some objections about the covariance of the equation has to be taken into account [29].

In 1960, before the gravitational self-force was proposed, DeWitt and Brehme [9] proposed an equation of motion for a charged particle which contains the electromagnetic self-force in a curved space. A few years later, Hobbs [10] corrected the equation by adding the so-called Ricci term. A very elegant and formal deduction of it can be found in the work done by Poisson et al. [17]. However, if we make an analysis of the Hobbs equation, as for example in Poisson et al. review [17], we will notice that, apart from the special notion of point charged particle, there is no special consideration on the metric tensor which describes the curved space. That is, the metric tensor corresponds to the one generated by all the particles including the charged particle itself. This means that Equation (62) holds and we just need to substitute the expression of the self-force and the metric tensor has to be considered as $\mathrm{d} \tilde{\tau}=-\tilde{g}_{\mu \nu} \mathrm{d} z^{\mu} \mathrm{d} z^{v}$.

It has to be noted that $\gamma^{\alpha \beta}$ has been calculated by using the average technique. Therefore, the proper way to express the terms $\gamma^{\alpha \beta}$ or $h^{\alpha \beta}$ should be with some sign that shows the average value; that is, $\hat{\gamma}^{\alpha \beta}$ and $\bar{h}^{\alpha \beta}$. This means that Equation (65) must be substituted by the average metric tensor

$$
\hat{g}_{\mu \nu}=g_{\mu v}+\widehat{h}_{\mu \nu},
$$

which can be considered as the effective metric tensor at the world line of the particle and the proper time must be expressed as an effective proper time

$$
\mathrm{d} \widehat{\tau}=-\widehat{g}_{\mu \nu} \mathrm{d} z^{\mu} \mathrm{d} z^{v}
$$

In the case of a single small point massive particle, It has to be also mentioned that the equation of motion is obtained by considering that the proper time represents an affine parameter of the geodesic. However, in the general case, due to the presence of electromagnetic forces the proper time is not anymore an affine parameter and therefore the expression for the acceleration is not so simple. The generalization of the Hobbs equation for a system of small point massive charges, considering the gravitational self-force, may be written as:

$$
\begin{aligned}
& m_{i} \frac{D}{d \widehat{\tau}_{i}}\left[\frac{\mathrm{d} z_{i \mu}}{\mathrm{d} \bar{\tau}_{i}}\right]=q_{i} \sum_{j \neq i}^{n} F_{j \mu \nu} \frac{\mathrm{d} z_{i}^{v}}{\mathrm{~d} \widehat{\tau}_{i}}+q_{i} \widehat{F}_{i \mu \nu} \frac{\mathrm{d} z_{i}^{v}}{\mathrm{~d} \widehat{\tau}_{i}}=q_{i} \sum_{j \neq i}^{n} F_{j \mu \nu} \frac{\mathrm{d} z_{i}^{v}}{\mathrm{~d} \widehat{\tau}_{i}} \\
& +q_{i}^{2}\left(\widehat{g}_{\mu \nu}+\frac{\mathrm{d} z_{i \mu}}{\mathrm{d} \widehat{\tau}_{i}} \frac{\mathrm{d} z_{i v}}{\mathrm{~d} \bar{\tau}_{i}}\right)\left(\frac{2}{3} \frac{D}{d \widehat{\tau}_{i}}\left[\frac{D}{d \widehat{\tau}_{i}}\left[\frac{\mathrm{d} z_{i}^{v}}{\mathrm{~d} \widehat{\tau}_{i}}\right]\right]+\frac{1}{3} \widehat{R}_{\lambda}^{v} \frac{\mathrm{d} z_{i}^{\lambda}}{\mathrm{d} \widehat{\tau}_{i}}\right)+q_{i} \widehat{F}_{\mu \nu}^{\text {tail }} \frac{\mathrm{d} z_{i}^{v}}{\mathrm{~d} \widehat{\tau}_{i}} .
\end{aligned}
$$

It has to be noticed that even for non-charged particles the calculation of the metric tensor $\tilde{g}_{\mu v}$ is difficult but apparently possible [17], in the case of charged particles the Einstein field equations are always coupled with the electromagnetic energy-momentum tensor (Equation (64)) and the calculation seems to be impossible in the general case. On the other side, by using the Landau-Lifshitz [23] equation in Special Relativity (method that has been formally explained by Spohn [3] and physically by Rohrlich [4], [5]), Quinn and Wald [20] have proposed an equation which would represent the Landau-Lifshitz equation in curved space. This equation can be expressed now as

$$
m_{i} \frac{D}{d \widehat{\tau}_{i}}\left[\frac{\mathrm{d} z_{i \mu}}{\mathrm{d} \widehat{\tau}_{i}}\right]=q_{i} \sum_{j \neq i}^{n} F_{j \mu \nu} \frac{\mathrm{d} z_{i}^{v}}{\mathrm{~d} \widehat{\tau}_{i}}+q_{i}^{2}\left(\hat{g}_{\mu \nu}+\frac{\mathrm{d} z_{i \mu}}{\mathrm{d} \widehat{\tau}_{i}} \frac{\mathrm{d}}{i v} \widehat{\tau}_{i}\right)\left(\frac{2}{3 m} \frac{D}{\mathrm{~d} \widehat{\tau}_{i}}\left[q_{i} \sum_{j \neq i}^{n} F_{j \mu \nu} \frac{\mathrm{d} z_{i}^{v}}{\mathrm{~d} \widehat{\tau}_{i}}\right]+\frac{1}{3} \widehat{R}_{\lambda}^{v} \frac{\mathrm{d} z_{i}^{\lambda}}{\mathrm{d} \bar{\tau}_{i}}\right)+q_{i} \widehat{F}_{\mu \nu}^{t a i l} \frac{\mathrm{d} z_{i}^{v}}{\mathrm{~d} \bar{\tau}_{i}} .
$$

\section{Concluding Remarks}

There are three lines in order to close the problem: the first one consists of clarifying the doubts about the nonunicity of the self-actions starting by the different equations in Special Relativity [2]; secondly, it is necessary to justify that the deduction of the self-forces is covariant taking into account the arguments related with illegitimate mathematical operation of summing vectors in different tangent spaces [28], and with the possible non-generally covariance of the MiSaTaQuWa equation which apparently reflects a specific choice of coordinate system and does not preserve its form under an infinitesimal coordinate transformation [29]; finally, the calculation of the metric tensor $\hat{g}_{\mu \nu}$ and the tail term in the general case of charged particles have to be improved. It has to be noticed that maybe the key to solve the problem belongs to the knowledge of the internal structure of the point 
charged particles (an important effort has been done in the last years [17]). Also it has to be mentioned that the deductions of the trajectories for different cases have contributed to understand the physics of the problem [35]-[37]. A comment about the tail terms has to be done. It is clear that the existences of the tail terms contribute to the non-applicability of the Stronger Equivalence Principle for charged particles but also for non-charged particles. However, in Quantum Mechanics, the Pauli Exclusion Principle (quantum indistinguishability) is not compatible with the tail terms since the motion of each particle will depend on its history and this will permit to distinguish each particle from the others. This contradiction has to be explained.

The main result of this article is that it has been shown that from a complete action the Einstein field equations, the Maxwell equations and the equations of motions including the self-actions can be deduced. In these orders of ideas, the formal inconsistency of the theory, commented by Hammond [23], partially disappears since even if the existences of the self-actions have been derived from an action principle, the deductions of their explicit forms are based on different anzats that generate, as we have mentioned, many discussions about their validities [2] [11]-[13] [28] [29].

\section{Acknowledgements}

This work was partially supported by C.O.F.A.A and E.D.I., I.P.N.

\section{References}

[1] Dirac, P.M. (1938) Proceedings of the Royal Society of London A, 167, 148-169. http://dx.doi.org/10.1098/rspa.1938.0124

[2] Parrot, S. (1987) Relativistic Electrodynamics and Differential Geometry. Springer, New York. http://dx.doi.org/10.1007/978-1-4612-4684-8

[3] Spohn, H. (2000) Europhysics Letters, 50, 287-292. http://dx.doi.org/10.1209/epl/i2000-00268-x

[4] Rohrlich, F. (2000) American Journal of Physics, 68, 1109 -1113. http://dx.doi.org/10.1119/1.1286430

[5] Rohrlich, F. (2001) Physics Letters A, 283, 276-278. http://dx.doi.org/10.1016/S0375-9601(01)00264-X

[6] Rohrlich, F. (2007) Classical Charged Particles. Word Scientific Publishing Co., Danvers. http://dx.doi.org/10.1142/6220

[7] Ares de Parga, G. (2006) Foundations of Physics, 36, 1474-1510. http://dx.doi.org/10.1007/s10701-006-9072-x

[8] Yaghjian, A.D. (1992) Relativistic Dynamics of a Charged Sphere. Springer, New York.

[9] DeWitt, B.S. and Brehme, R.W. (1960) Annals of Physics, 9, 220-259. http://dx.doi.org/10.1016/0003-4916(60)90030-0

[10] Hobbs, J.M. (1968) Annals of Physics, 47, 141-165. http://dx.doi.org/10.1016/0003-4916(68)90231-5

[11] Boulware, D. (1980) Annals of Physics, 124, 169-188. http://dx.doi.org/10.1016/0003-4916(80)90360-7

[12] Singal, A.K. (1995) General Relativity and Gravitation, 27, 953-967. http://dx.doi.org/10.1007/BF02113077

[13] Parrot, S. (2002) Foundations of Physics, 32, 407-440. http://dx.doi.org/10.1023/A:1014861329235

[14] López-Bonilla, J.L. Morales, J. and Rosales, M.A. (1994) Pramana: Journal of Physics, 43, 273-278. http://dx.doi.org/10.1007/BF02846843

[15] Teitelboim, C. (1971) Physical Review D, 4, 345-347. http://dx.doi.org/10.1103/PhysRevD.4.345

[16] Plebañski, J. (1972) The Structure of the Field of a Point Charges. Internal report CINVESTAV-IPN, Mexico.

[17] Poisson, E., Pound, A. and Vega, I. (2011) Living Reviews in Relativity, 14, 1-190. http://dx.doi.org/10.12942/1rr-2011-7

[18] Hadamard, J. (1923) Lectures on Cauchy's Problem in Linear Partial Differential Equations. Yale University Press, New Haven.

[19] Detweiler, S. and Whiting, B.F. (2003) Physical Review D, 67, Article ID: 024025. http://dx.doi.org/10.1103/PhysRevD.67.024025

[20] Quinn, T.C. and Wald, R.M. (1997) Physical Review D, 56, 3381-3394. http://dx.doi.org/10.1103/PhysRevD.56.3381

[21] Mino, Y., Sasaki, M. and Tanaka, T. (1997) Physical Review D, 55, 3457-3476. http://dx.doi.org/10.1103/PhysRevD.55.3457

[22] Ghosh, S., Choudhury, A. and Sarma, J.K. (2013) Indian Journal of Physics, 87, 607-611. http://dx.doi.org/10.1007/s12648-013-0254-Z 
[23] Landau, L.D. and Lifshitz, E.M. (1962) The Classical Theory of Fields. Pergamon, London.

[24] Weinberg, S. (1972) Gravitation and Cosmology: Principles and Applications of the General Theory of Relativity. John Wiley \& Sons, New York.

[25] Hammond, R.T. (2010) EJTP, 7, 221-258.

[26] Tessarotto, M., Dorigo, M., Cremaschinid, C., Nicolinia, P. and Beklemishev, A. (2008) The Exact Radiation-Reaction Equation for a Classical Charged Particle. arXiv:0807.1819v1.

[27] Galey, C.R., Hu, B.L. and Lin, S.Y. (2006) Physical Review D, 74, Article ID: 024017. http://dx.doi.org/10.1103/PhysRevD.74.024017

[28] Parrot, S. (2003) Energy Radiation of Charged Particles in Conformally Flat Spacetimes. arXiv:gr-qc/9308023v3.

[29] Kim, D.H. (2005) Radiation Reaction in Curved Spacetime. Ph.D. Thesis, University of Florida, Gainesville.

[30] Geroch, R. and Traschen, J. (1987) Physical Review D, 36, 1017-1031. http://dx.doi.org/10.1103/PhysRevD.36.1017

[31] McGregor, M.H. (1992) The Enigmatic Electron. Kluer Academic Publishers, Dordrecht.

[32] Barut, A.O. and Zanghi, N. (1984) Physical Review Letters, 52, 2009-2012. http://dx.doi.org/10.1103/PhysRevLett.52.2009

[33] Ghosh, S., Choudhury, A. and Sarma, J.K. (2012) Apeiron, 19, 247-263.

[34] Ghosh, S., Choudhury, A. and Sarma, J.K. (2012) Indian Journal of Physics, 86, 481-483. http://dx.doi.org/10.1007/s12648-012-0083-5

[35] Barack, L. (2000) Physical Review D, 62, Article ID: 084027. http://dx.doi.org/10.1103/PhysRevD.62.084027

[36] Pound, A. and Poisson, E. (2008) Physical Review D, 77, Article ID: 044013. http://dx.doi.org/10.1103/PhysRevD.77.044013

[37] Vega, I., Wardell, B. and Diener, P. (2011) Classical and Quantum Gravity, 28, Article ID: 134010. http://dx.doi.org/10.1088/0264-9381/28/13/134010

[38] Gal'tsov, D.V. and Spirin, P.A. (2008) Gravitational Radiation Reaction in Non-Vacuum Space-Time. CAPRA Meeting, Orleans, France, 1-21.

[39] Messaritaki, E. (2003) Radiation Reaction on Moving Particles in General Relativity. Ph.D. Thesis, University of Florida, Gainesville.

[40] Detweiler, S. (2001) Physical Review Letters, 86, 1931-1934. http://dx.doi.org/10.1103/PhysRevLett.86.1931 